\title{
Whole genome sequencing of Ethiopian highlanders reveals conserved hypoxia tolerance genes
}

\author{
Nitin Udpa ${ }^{1 \dagger}$, Roy Ronen ${ }^{1 \dagger}$, Dan Zhou ${ }^{2 \dagger}$, Junbin Liang ${ }^{3}$, Tsering Stobdan ${ }^{2}$, Otto Appenzeller ${ }^{4}$, Ye Yin ${ }^{5}$, \\ Yuanping Dư ${ }^{5}$, Lixia Guo ${ }^{3}$, Rui $\mathrm{CaO}^{3}$, Yu Wang ${ }^{5}$, Xin Jin ${ }^{3}$, Chen Huang ${ }^{5}$, Wenlong Jia ${ }^{3}$, Dandan Cao ${ }^{3}$, \\ Guangwu Guo ${ }^{3}$, Victoria E Claydon ${ }^{6}$, Roger Hainsworth', Jorge L Gamboa ${ }^{8}$, Mehila Zibenigus ${ }^{9}$, Guta Zenebe ${ }^{9}$, \\ Jin Xue ${ }^{2}$, Siqi Liu ${ }^{10}$, Kelly A Frazer ${ }^{11}$, Yingrui Li ${ }^{5}$, Vineet Bafna ${ }^{12}$ and Gabriel G Haddad ${ }^{2,13,14^{*}}$
}

\begin{abstract}
Background: Although it has long been proposed that genetic factors contribute to adaptation to high altitude, such factors remain largely unverified. Recent advances in high-throughput sequencing have made it feasible to analyze genome-wide patterns of genetic variation in human populations. Since traditionally such studies surveyed only a small fraction of the genome, interpretation of the results was limited.

Results: We report here the results of the first whole genome resequencing-based analysis identifying genes that likely modulate high altitude adaptation in native Ethiopians residing at 3,500 m above sea level on Bale Plateau or Chennek field in Ethiopia. Using cross-population tests of selection, we identify regions with a significant loss of diversity, indicative of a selective sweep. We focus on a $208 \mathrm{kbp}$ gene-rich region on chromosome 19, which is significant in both of the Ethiopian subpopulations sampled. This region contains eight protein-coding genes and spans 135 SNPs. To elucidate its potential role in hypoxia tolerance, we experimentally tested whether individual genes from the region affect hypoxia tolerance in Drosophila. Three genes significantly impact survival rates in low oxygen: cic, an ortholog of human CIC, HSI, an ortholog of human LIPE, and Paf-AHa, an ortholog of human PAFAH1B3.

Conclusions: Our study reveals evolutionarily conserved genes that modulate hypoxia tolerance. In addition, we show that many of our results would likely be unattainable using data from exome sequencing or microarray studies. This highlights the importance of whole genome sequencing for investigating adaptation by natural selection.
\end{abstract}

\section{Background}

Humans have occupied high altitude regions for thousands of years [1]. It is estimated that currently more than 140 million people live and work at altitudes above $2,500 \mathrm{~m}[2]$, where hypoxic conditions present a challenge for survival. Previous studies suggest that the three large high altitude populations (that is, Andeans, Himalayans, and Ethiopians) have each adapted uniquely to cope with their inhospitable hypoxic environments

\footnotetext{
* Correspondence: ghaddad@ucsd.edu

${ }^{\dagger}$ Equal contributors

${ }^{2}$ Department of Pediatrics, Division of Respiratory Medicine, University of California San Diego, La Jolla, California 92093, USA

${ }^{13}$ Department of Neurosciences, University of California San Diego, La Jolla, California 92093, USA

Full list of author information is available at the end of the article
}

$[3,4]$. It has also been suggested that the Ethiopians are better adapted to these conditions, as they show the least evidence of chronic mountain sickness (CMS), a high altitude syndrome that exists in other populations, especially the Andeans [5]. For instance, of the three highlander populations, Ethiopians show arterial oxygen levels that are most similar to sea level controls $[4,6]$. Although it has long been proposed that genetic factors contribute to adaptation to high altitude, these remain largely unproven [7]. Recent advances in high-throughput sequencing technologies have made it feasible to analyze patterns of genetic variation in human populations across the entire genome. To date, several genomic scans for natural selection have been performed in high altitude populations (for

\section{Biomed Central}


instance, [8-15]); however, as these studies either focused on a priori candidate genes, or assayed small portions of the genome (exons or a subset of genotyped SNPs), there is likely much yet to be deciphered.

We previously performed the first whole genome resequencing-based analysis of genes contributing to hypoxia adaptation in a tolerant Drosophila strain, generated through laboratory evolution [16]. Examining the complete genome of adapted populations, we were able to detect fine changes in the allele frequency spectrum consistent with natural selection. Extending the analytical strategy from our previous study, we present here the results of a whole genome resequencing-based analysis identifying genes that likely contribute to high altitude adaptation in humans. We focused our study on 13 high altitude (approximately 3,500 m) native Ethiopian residents. Specifically, we analyzed the genomes of six individuals of Oromo heritage living on Bale Plateau (labeled 'Oromos'), and seven individuals residing on the Chennek field in the Simien Mountains (labeled 'Amhara').

While our study uses well-known statistical tests to identify genomic regions undergoing a selective sweep, we have made a number of novel design choices compared with previous studies of human adaptation [8-15]. First, we use whole genome sequencing (WGS) rather than genotyping arrays, allowing a much richer sampling of the site frequency spectrum in a given region. Second, in order to test whether particular genes highlighted by our analysis play a role in hypoxia tolerance, we used RNA interference (RNAi) to target their respective orthologs in Drosophila melanogaster. Thus, in addition to reporting a set of regions showing a strong signature of positive selection in high altitude populations (as in previous studies), we also report on several genes that, apart from showing such a signature, are shown experimentally to modulate the adaptive phenotype.

\section{Results}

We sequenced the genome of each individual using Illumina's Hiseq 2000 platform to a mean genome-wide depth of approximately $18 \times$ per individual. We mapped the reads to the hg19 human reference using BWA [17], and performed variant calling using the GATK pipeline $[18,19]$. See Additional file 1 for an overview of the computational pipeline.

We then used ADMIXTURE [20] to identify the closest populations from the 1000 Genomes Project [21], release 20100804. This showed that our Ethiopian highlanders share common genetic ancestry, and are largely an admixture of two ancestral groups (Additional file 2). The largest ancestry component shows high similarity to African populations, particularly the Luhya (LWK), located in neighboring Kenya. The remainder is largely shared with individuals of non-Finnish, European ancestry. As a result, for lowlander controls, we used variant calls from low coverage whole-genome sequencing of 67 Luhya (LWK) individuals. As an out-group we used 90 northern European ancestry (CEU) individuals. We also performed principle component analysis (Additional file 3) on our study populations jointly with the lowlander controls (LWK) and outgroup (CEU), further illustrating our study populations as an admixture of these two ancestral groups. Due to differences in coverage between the control populations and our Ethiopian sequence data, we filtered low coverage or poor quality loci prior to testing for selection (see Materials and methods).

\section{Genome-wide scans of selection}

Under environmental selective stress, such as hypoxia, alleles that confer an adaptive advantage are likely to increase in frequency, along with their linked neighbors. This process is known as a 'selective sweep'. We sought regions with evidence of such a sweep: a loss of genetic diversity in the region and a corresponding decrease in the scaled mutation rate, $\theta\left(=4 N_{e} \mu\right.$ where $N_{e}$ is the effective population size and $\mu$ is the mutation rate). We computed four cross-population test statistics (denoted $\mathrm{S}_{\mathrm{f}}, \mathrm{S}_{\pi}, \mathrm{F}_{\mathrm{s}}$, and population branch statistic (PBS); see Materials and methods) that measure this loss in diversity. Cross-population tests provide a control for locusspecific variability in scaled mutation rates, enabling a direct comparison of the effective population size as a measure of selection $\left(\mathrm{S}_{\mathrm{f}}\right.$ and $\left.\mathrm{S}_{\pi}\right)$. They also allow for an estimation of branch lengths and bottlenecks relative to the point of divergence between populations $\left(\mathrm{F}_{\mathrm{st}}\right.$ and PBS). Through extensive simulations, we showed that the power of these tests varies depending on the selection coefficient and time since selection, among other things [22] (Materials and methods; Additional files 4 and 5). As these parameters are unknown, we considered regions that were significant under any of these tests.

We assume that the genetic basis for the adaptation to low oxygen influences relatively few loci genome-wide. As a result, for a cross-population test, the null distribution of two neutrally evolving populations can be approximated by the observed distribution of highlanders versus lowlander controls. We report regions exceeding the top $0.1 \%$ genome-wide value for each test. For the Amhara population, these values were 0.16 (PBS), 0.18 $\left(\mathrm{F}_{\mathrm{st}}\right), 1.73\left(\mathrm{~S}_{\pi}\right)$, and $2.0\left(\mathrm{~S}_{\mathrm{f}}\right)$. For the Oromos, these were $0.15(\mathrm{PBS}), 0.16\left(\mathrm{~F}_{\mathrm{st}}\right), 1.61\left(\mathrm{~S}_{\mathrm{\pi}}\right)$, and $1.88\left(\mathrm{~S}_{\mathrm{f}}\right)$.

We initially identified 420 regions spanning $36.8 \mathrm{Mbp}$ as significant in at least one test under the corresponding $0.1 \%$ genome-wide false discovery rate (FDR; see Additional file 6 for a summary, and Additional file 7 for the complete set of regions identified by the four tests). While genome-wide scans for selection are a powerful tool for detecting genetic factors contributing to adaptation, it 
is also true that these scans make no guarantees on the significance of the results $[23,24]$. Consequently, we provide experimental evidence to further support the role of some of our highlighted genes in hypoxia adaptation. Due to the infeasibility of doing this for all genes identified by our tests, we were faced with the need to prioritize candidate regions that showed the strongest evidence of testable selection, and that appeared unique to Ethiopian highlanders. We thus implemented a series of automated prioritization criteria.

\section{Region prioritization}

\section{Frequency block differential relative to lowlander control}

A region under strong positive selection is characterized by changes in allele frequencies that cannot be explained by a neutral model. These often manifest as blocks of SNPs with increased minor allele frequency. We leverage this fact by seeking regions with multiple SNPs present in a block structure, at comparatively high frequencies in the highlander populations. Given a population sample of size $n$, we iterate over all possible frequency values $f$, where $f=(1 / n, 2 / n, \ldots,(n-1) / n)$. For each value of $f$, we isolate the variants in the region of frequency within $1 / n$ from $f$. From these, we define a $f$-frequency block as a subset of $\geq 10$ consecutive SNPs. Then, for each such block, we calculate the frequency differential, defined as the absolute difference in mean frequency between the study population and the closer of the LWK and CEU lowlander controls. We focus on regions with large block differential. Specifically, we consider only regions with block differential exceeding the 95\% confidence interval (CI) of the sampling variance when sampling $n=12$ (for Oromos) or $n=14$ (for Amhara) haplotypes from a population (roughly 20\%; see Discussion; Additional file 8). Fifty-eight regions were prioritized after this step.

\section{Frequency block differential relative to HapMap control populations}

To ensure that the prioritized regions represent positive selection only in Amhara or Oromos highlanders, we expanded our controls to include additional lowlander populations. Specifically, we prioritized regions with block differential exceeding the $95 \% \mathrm{CI}$ of the sampling variance (see above) compared to all HapMap populations [25]. This ensures we do not consider regions where the dominant haplotype block exists at similar frequency in lowlander populations. Specifically, this helps to avoid spurious signal of selection due to nonAfrican admixture, but also signal that is common between the highlanders and other lowlander populations. Apart from admixture, such common regions may come about independently (due to similar selective constraints), or may be the remnants of selection in an ancestral population. Twenty-seven regions remained prioritized after this step.

\section{Frequency block differential after integrating existing genotype data}

We also used variant calls from a previous study by Alkorta-Aranburu et al. [14] on the same highlander populations. In this study, the authors performed genotyping on 102 Amhara highlanders, and 63 Oromos highlanders. By incorporating the allele frequencies observed in genotyping these larger cohorts, we were able to refine our sample frequencies and identify any false signals that were caused by sampling. For a given region, we extracted all variants from the (previously identified) $f$-frequency block, which were also covered by AlkortaAranburu et al. We then refined our highlander (Amhara or Oromos) block frequency by taking a weighted average (by sample size) over the observed frequencies in the corresponding population from both studies. For instance, if the observed mean frequency for a haplotype block was 0.8 in our Oromo sample $(n=12)$, and 0.85 in the Oromo sample from Alkorta-Aranburu et al. $(n=$ 126), the revised block frequency would be set to 0.846 . Due to the increased sample size, the $95 \%$ CI of sampling error was reduced substantially. Consequently, we prioritized regions where the revised block frequency differential was greater than $10 \%$ for all controls. We note that regions that contained no variants sampled by AlkortaAranburu et al. were unaffected by this criterion. Nineteen regions remained prioritized after this step.

\section{RefSeq genes in region}

Finally, we prioritized candidate regions that overlap at least one transcript, as defined by RefSeq (release 45, downloaded 14 January 2011). This collection includes protein coding genes, microRNAs, and non-coding RNAs (39,173 transcripts overall). Other regions may contain important regulatory variations; however, for an initial pass, we focused our efforts on regions for which there are more readily accessible methods to identify and validate causal effects.

\section{Prioritized regions}

At the end of this process, only eight regions remained in our list of prioritized regions (see Table 1 for a summary; see Additional file 9 for Manhattan plots). Of these, two were significant in both the Amhara and the Oromos populations. However, due to a lack of overlapping sites from HapMap [25] or from the AlkortaAranburu et al. study, we were unable to subject one of these loci (chr14:106.32-106.39 M) to the complete battery of prioritization criteria. As a result, we focused on the remaining shared region.

This $208 \mathrm{kbp}$ gene-rich region on chromosome 19 contains a block of 135 'differential' SNPs showing significant 
Table 1 Significant genomic regions in the Amhara and/or the Oromos populations

\begin{tabular}{llllll}
\hline & Chromosomal region & A & $\mathbf{0}$ & Tests & Genes located in the region \\
\hline 1 & Chr6:29796452-29896452 & & $\checkmark$ & $\mathrm{S}_{\pi}, \mathrm{S}_{\mathrm{f}}$ & HLA-G, HLA-H, HCG2P7, HCG4P6 \\
2 & Chr9:33915871-34021871 & & $\checkmark$ & $\mathrm{S}_{\pi}, \mathrm{S}_{\mathrm{f}}$ & UBE2R2, UBAP2, SNORD121ANB \\
3 & Chr11:84676260-84910260 & $\checkmark$ & & $\mathrm{S}_{\pi,} \mathrm{S}_{\mathrm{f}}$ & DLG2 \\
4 & Chr13:78496785-78606785 & $\checkmark$ & $\mathrm{S}_{\pi}$ & EDNRB \\
5 & Chr14:106322845-106396845 & $\checkmark$ & $\checkmark$ & $\mathrm{F}_{\mathrm{st}}$ & KIAA0125 \\
6 & Chr19:42741726-42973726 & $\checkmark$ & $\checkmark$ & PBS & GSK3A, ERF, CIC*, PAFAH1B3*, PRR19, \\
7 & & & & & TMEM145, MEGF8, CNFN, LIPE*, CXCL17 \\
8 & ChrX:44982060-45036060 & & $\checkmark$ & $\mathrm{S}_{\pi}, \mathrm{S}_{\mathrm{f}}$ & CXorf36 \\
\hline
\end{tabular}

*Genes experimentally validated as affecting hypoxia tolerance in Drosophila.

change in frequency relative to the control populations (Figure 1). Specifically, the mean variant frequencies are 48\% (Oromos), 42\% (Amhara), 16\% (LWK), and 1\% (CEU). We computed the local linkage disequilibrium (LD) structure in the region, and found a strong, and very large, LD block surrounding the region in the Oromos (Additional file 10). A corresponding, but smaller, block was also visible in the Amhara. This is consistent with the longer timespan spent at high altitudes by the Amhara [26], during which recombination may have broken local LD structure.
Of the 10 genes in the region (Table 1), 8 lie in the prioritized region shared across the Oromos and the Amhara. These genes point to many intriguing candidates. For example, the differential SNPs include two missense mutations in the LIPE gene (rs7246232 and rs16975750; Additional file 11). While these mutations have not previously been linked to a known phenotype, LIPE is associated with gestational hypertension (and consequent placental ischemia) [27]. It belongs to the lipase family, which is known to play a role in hypoxia via lipolysis, triglyceride metabolism, and energy storage [28]. Other
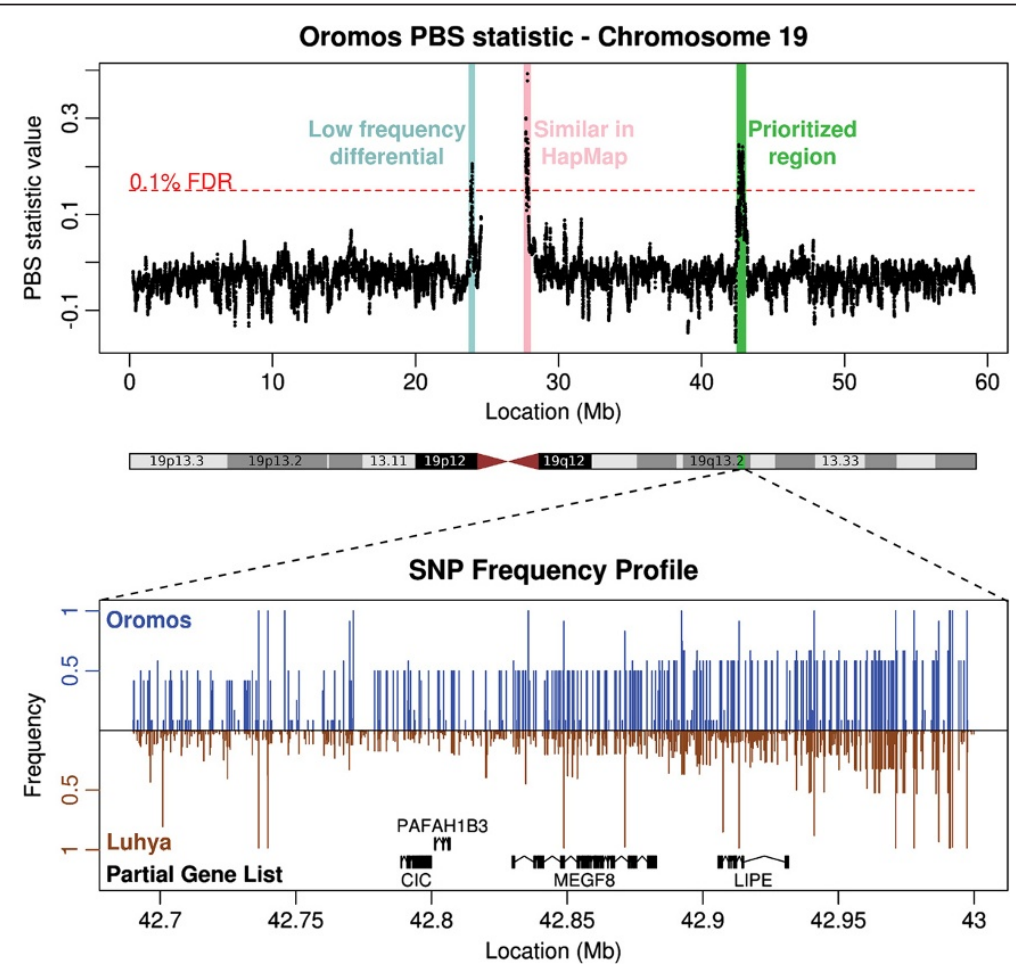

Figure 1 Population branch statistic (PBS) across chromosome 19 in the Oromos population compared to both the Luhya (LWK) and European (CEU) populations. The red line represents a genome-wide $0.1 \%$ FDR. Three distinct regions exceed this cutoff, two of which are near the centromere and were thus not prioritized. The bottom panel shows the SNP frequency profile in the prioritized region for Oromos (blue) and LWK (brown, inverted). Genes with Drosophila orthologs (on which RNAi experiments were conducted) are shown in black below the frequency profiles. As can be seen, variant frequencies in this region are considerably higher in the highlanders than in a nearby lowlander population. 
genes in this region include $C I C$, which is a transcriptional suppressor involved in early organ development, CNFN (involved in hematopoiesis [29]), CXCL17 (involved in angiogenesis [30]), and PAFAH1B3 (related to coronary artery disease [31] and organ development [32]). Thus, our results point to a cluster of putative hypoxia response genes. As these genes are associated with phenotypes such as lipid metabolism, transcription regulation, or angiogenesis, they illustrate the potential for a variety of adaptive mechanisms to high altitude in humans.

The remaining seven regions (Table 1) contained several other intriguing gene candidates. For instance, the 110 kbp region on chromosome 13 that is significant for the Amhara population under the $S_{\pi}$ test contains Endothelin receptor $\mathrm{B}$ (EDNRB; Additional file 12). This gene encodes a receptor for endothelin, a potent vasoactive peptide, which activates signaling cascades that promote blood vessel constriction [33]. EDNRB is also tied into the HIF pathway. Specifically, it is a receptor for Endothelin 1, which is directly activated by HIF. In addition, it is a known target for drugs (for example, bosentan) prescribed for altitude sickness [34]. In the Amhara population, this gene has 52 fixed, or near-fixed, SNPs (spanning approximately $170 \mathrm{kbp}$ ) upstream of the promoter region, 20 of which are in a $10 \mathrm{kbp}$ region containing several transcription-factor binding sites (Additional file 7). As a result, and due to the lack of nonsynonymous coding mutations, we hypothesize that adaptive effects in this region are likely due to regulatory variation. Further study will be needed to determine the mechanism by which this may have occurred. Additionally, we note that the dominant haplotype block is present in the controls, at 36\% frequency in LWK and 66\% frequency in CEU. Such intermediate frequencies in lowlander controls are consistent with selection acting on standing variation, rather than a de novo mutation [35].

\section{Experimental validation using a model system}

To provide further evidence of the role of these genes in hypoxia, we used D. melanogaster as a model system to test the hypothesis that differential regulation of their orthologs in flies affects tolerance or susceptibility to low $\mathrm{O}_{2}$. Potentially causal variants in the candidate genes may represent either gain- or loss-of-function changes. Due to a lack of nonsynonymous coding variants in most of the genes (with the exception of LIPE), we hypothesize that adaptive traits are likely the result of regulatory effects.

Because up-regulating a gene may be problematic if it is unexpressed in a particular tissue, we first used the UAS-RNAi/GAL4 system (see Materials and methods) to investigate whether down-regulating the fly orthologs of the candidate genes in the chromosome 19 region has any effect on hypoxia tolerance. Of the eight genes in the region, we tested four (CIC, LIPE, PAFAH1B3, and
MEGF8) that had Drosophila orthologs. Remarkably, three of the four genes, when knocked down, led to markedly improved tolerance to low oxygen. These genes were cic (ortholog of human CIC), Hsl (ortholog

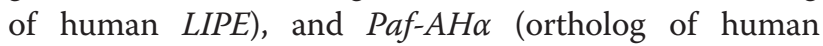
PAFAH1B3). We observed an increase in survival rates that varied from about $40 \%$ to $80 \%$, constituting a twoto four-fold increase over controls in the same hypoxic environment (Figure 2).

In contrast, we recently tested 2,187 P-elements fly lines covering 1,870 genes [36] and obtained only 44 lines (approximately 1.5\%) showing evidence of tolerance to low $\mathrm{O}_{2}$. Assuming this roughly represents the null distribution of random genes affecting hypoxia tolerance, our observation of three of the four tested genes with this effect is statistically significant $\left(P=3.7 \times 10^{-4}\right.$, Fisher's exact test).

We note that $\mathrm{CIC}$ has been shown to function as a repressor of receptor tyrosine kinase (RTK) responsive genes. Following activation of RTK signaling, CIC repression is removed, enabling expression of targeted genes downstream. CIC is well conserved from Drosophila to humans, and is mostly known in determining cell fate and cell proliferation $[37,38]$. Of interest is the

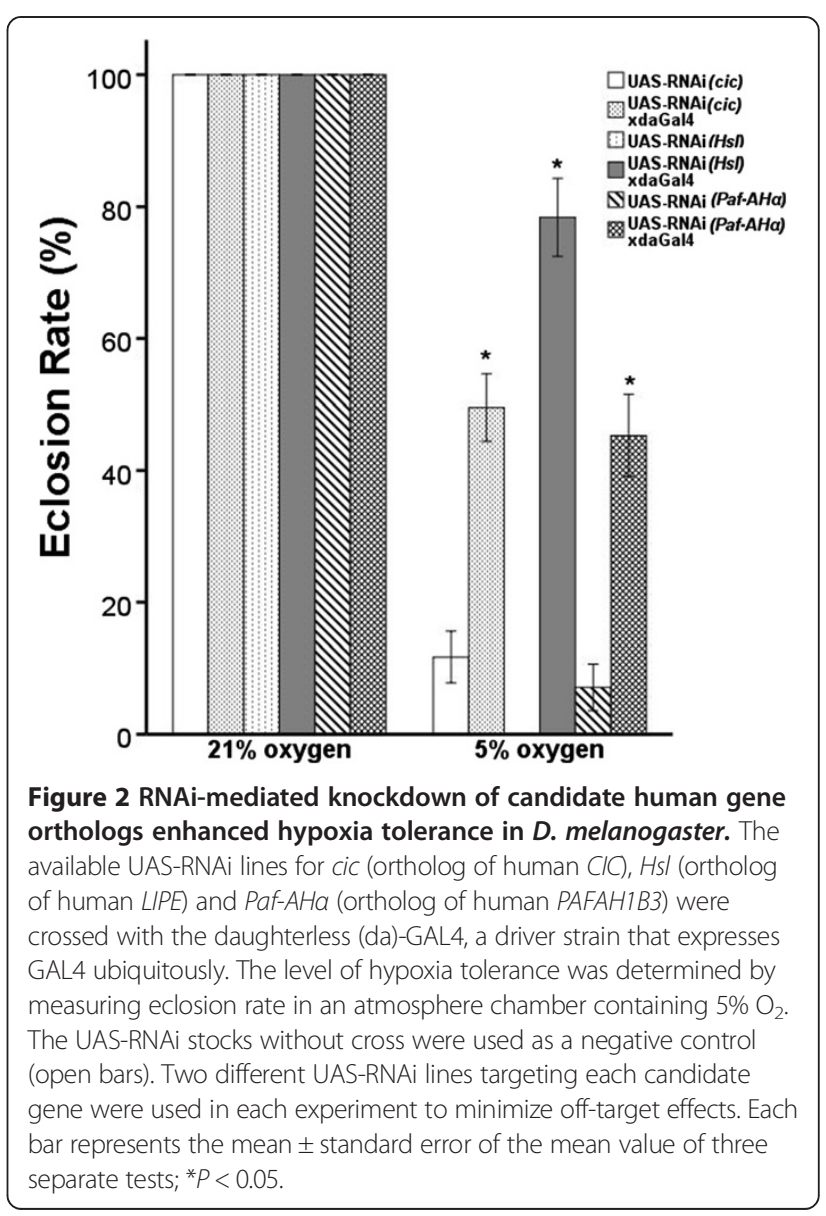


cross-talk between the RTK and Notch pathways, including core components of the RTK pathway and other major pathways such as transforming growth factor beta, Jak/Stat and Wnt [39]. This is remarkable, as we have previously shown the Notch pathway to be crucial for hypoxia tolerance in Drosophila [16]. LIPE, a hormonesensitive lipase, is important in lipolysis and in mobilization of fatty acids and glycerol from fat cells. PAF, a plateletactivating factor, is a potent lipid mediator and is involved in a variety of physiological events. Its deacetylation induces a loss of activity that is catalyzed by PAF-AH, a plateletactivating factor acetyl hydrolase. Type I PAF-AH has two subunits $(\alpha$ and $\beta)$ and plays a role in cellular functions such as induction of nuclear movement and control of microtubule organization. Further study will be required in order to determine how exactly reduced expression of these transcripts contributes to improved hypoxia tolerance.

Despite other phenotypic differences (see 'Sample description' in Materials and methods), the Amhara and Oromos are both well adapted to high altitudes. Nevertheless, as evident from the chronic mountain sickness scores (Additional file 13), the Amhara appear to be somewhat better adapted. This is consistent with the longer time spent in high altitudes, allowing more opportunity for adaptation to occur. The comparatively shorter time of the Oromos at altitude (600 to 700 years [26]) implies rapid adaptation. One possible explanation for this is selection acting on standing variation, rather than on de novo mutations [35]. If true, this may also explain commonalities in adaptation between the populations, as in the chromosome 19 region. An alternative explanation is that a beneficial allele arose in one of the populations, and early migration/ admixture carried the haplotype block to the other.

We emphasize that despite this shared region, the majority of the signal for selection is not shared between the two populations (Table 1; Additional file 7). As hypoxia resistance is likely a systematic, complex, and multi-genic trait, we believe that the genes uncovered here explain only part of the adaptive trait, and that further studies in both populations will be required to fully elucidate the adaptive mechanism.

\section{Discussion}

Although the notion that hypoxia tolerance is heritable has dominated high altitude medicine for some time, it was only in recent years that attempts to identify the genetic basis of this adaptation have been made. These studies used genotyping or exome sequencing, but not WGS. The relatively sparse sampling of the genome obtained with these technologies makes it harder to identify shifts in the allele frequency spectrum associated with natural selection. Consequently, many of these studies focused on candidate genes. Moore et al. [9] reported one of the first genomic scans for selection in high altitude human populations. Considering roughly 11,000 SNPs, they identified variants in genes related to the hypoxia inducible factor (HIF) pathway, such as Endothelin 1. An extended analysis using genotyping arrays [40] identified candidate genes related to the HIF pathway, including ETA, NOS $2 A$, and PRKAA1. Another study by Simonson et al. [11] used LD-based tests, finding signatures of selection in EGLN1 and PPAR $\alpha$. Beall et al. [12] identified positive selection in a sample of high-altitude Tibetans at the EPAS1 locus. Similar studies were also carried out in Ethiopia by Scheinfeldt et al. [13], identifying CBARA1, VAV3, ARNT2 and THRB (the latter two are related to the HIF pathway), by AlkortaAranburu et al. [14], identifying several hypoxia-related genes (for example, CUL3, ADRBK1, CORO1B), and most recently by Huerta-Sanchez et al. [15], identifying a HIF-related gene (BHLHE41). Interestingly, we did not observe strong signals of positive selection in our Ethiopian populations for any of these genes. This may be the result of a different assaying technique, as this is the first study in Ethiopians to use WGS in a genome-wide scan for selection. We note that the above-mentioned studies showed no experimental evidence supporting the role of the identified genes in hypoxia tolerance.

There is an important trade-off when comparing WGS to exome sequencing or genotyping studies. Namely, WGS is usually performed on fewer individuals, but provides a near-complete sampling of variant sites. For selection signatures, this is critical. For instance, consider the high frequency block found near the EDNRB gene. With WGS, this region corresponds to the highest peak in the chromosome, with a block of 52 variants that are fixed in Amhara, but only 36\% in LWK. In contrast, the Nimblegen (Madison, WI, USA) $2.1 \mathrm{M}$ exon capture array targets only two high-frequency variants in this region, none within the block. As for genotyping, the approximately $1 \mathrm{M}$ Affymetrix (Santa Clara, CA, USA) Genome-Wide Human SNP Array 6.0 samples only 2 of the 52 sites in the block, resulting in a much weaker signal (Additional file 14). In both cases, signal in the region did not exceed the respective genome-wide $0.1 \%$ FDR, calculated using only the corresponding (exome or genotyping) sites (see Materials and methods). A similar argument holds for seven of the eight final regions (Table 1) identified in our study.

In addition, although genotype imputation is powerful for inferring un-sampled sites, it relies on conserved LD structure between the study population and a reference panel. Yet, positive selection strongly affects the structure of LD in a region by extending haplotype boundaries [41], as is evidently the case in the chromosome 19 region (Additional file 10). As a result, even if LD is generally well conserved between a study population and reference panel, this is less likely to hold in regions 
affected by positive selection, rendering imputation less effective in genomic scans for selection.

The drawback of sampling fewer individuals is that observed frequency differences may arise from sampling. To account for this, we determined the sampling variance $(95 \% \mathrm{CI})$ of a SNP at a given frequency that can be expected when sampling 12 to 14 haplotypes from a population (Additional file 8). We then prioritized regions showing a frequency differential that exceeded the sampling variance between highlanders and lowlander controls. Despite this limitation, simulations show that our tests achieve between 67 and 95\% power on 12 to 14 haplotypes, compared to a much larger sample of 400 haplotypes (Additional file 15). At the same time, many of the regions we identified would likely have been missed by genotyping studies, implying that WGS represents a complementary approach to sampling-based assays. An optimal study design may, for instance, include WGS of relatively few individuals, followed by targeted sequencing or genotyping of a larger cohort. This would enable a complete genomic scan, as well as increased power derived from larger samples. We achieved a similar design by integrating our WGS data with genotype data from Alkorta-Aranburu et al. [14].

\section{Conclusions}

Our study identifies a number of candidate genes for hypoxia tolerance that were not previously reported. To further validate our approach, we tested the impact of down-regulating these genes (using RNAi knock down) on hypoxia tolerance in a D. melanogaster model system. Several orthologs, when knocked down, led to increased survival (that is, eclosion) under low $\mathrm{O}_{2}$ conditions (two- to four-fold relative to controls). This provides evidence for their important role under hypoxic conditions, and lends further credence to our analysis. Finally, the fact that genes identified by WGS in humans affect flies implies an evolutionarily conserved mechanism for hypoxia tolerance.

\section{Materials and methods \\ Sample description}

Ten Oromos subjects from the Bale Plateau in the Oromia region of southeast Ethiopia and seven Amhara subjects from the Chennek field in the Simien Mountains of north Ethiopia were chosen to reflect differences in ancestral adaptation to high altitude. The Oromos generally have darker skin color and a less slender build. They appear more muscular and are generally shorter than the Amhara people. They have lived at high altitudes for 600 to 700 years; a much shorter time compared to the millennia of the Amhara people [26]. The subjects were examined and a history was taken. Only males aged 20 to 40 years found to be free of disease and with a chronic mountain sickness (CMS) score $<12$ [42] were selected. See Additional file 13 for the complete clinical characteristics of each test subject. Venous blood was obtained in the field, stored, and transported in suitable containers to allow extraction of sufficient DNA from both study populations. Subjects were volunteers, and each subject gave informed written consent in their local language, adhering to a protocol approved by the UCSD institutional review board (IRB00000354).

\section{DNA extraction, library construction and sequencing}

Genomic DNA was isolated using Blood DNA extraction kit (QIAGEN, Valencia, CA, USA) and randomly fragmented. Fragments of the desired length were gelpurified. Adapter ligation and DNA cluster preparation were performed using the library preparation kit according to manufacturer's instruction (Illumina, San Diego, CA, USA). Whole genome sequencing was performed using Illumina's Hiseq 2000 platform on all individuals to a mean, per-sample depth of approximately $18 \times$ (Additional file 16).

\section{Read alignment, score recalibration and variant calling}

We aligned the reads to the human reference genome (hg19) using BWA [17] with default parameter settings. We adjusted the alignments using GATK indel realignment, Picard read duplicate marking, and GATK quality score recalibration modules $[18,19]$ under default parameter settings, as defined by the GATK manual (version 2). We finally called and filtered the SNPs using the GATK UnifiedGenotyper tool under default settings. As can be seen in Additional file 16, the sequencing was free of any mapping bias in coverage or mapping percentage. As an independent test, we also identified variants using the SoapSNP pipeline [43]. The SoapSNP variants were generally a super-set of the GATK variants, with $25 \%$ more calls $(9,508,898$ versus $7,594,936$ for Amhara, and 10,284,853 versus $8,144,023$ for Oromos). This is mainly attributed to less restrictive filtering.

\section{Variant filters}

The coverage difference between our study populations (approximately $20 \times$ ) and lowlander controls (approximately $4 \times$ ) led to differences in processing the called variants. To adjust for these differences, we filtered our call set using three steps. First, we observed several variants in clustered genomic loci that were discarded by the variant caller in the study (higher coverage) populations. This happens due to various sequencing and mapping artifacts, such as strand bias, low sequence complexity, or structural variations. Due to the low coverage, variants in these loci are not always discarded in the controls. We thus removed from consideration any region comprising 10 consecutive SNPs that were filtered out using GATK in our study 
population. Second, following the protocol used by the 1000 Genomes Project, we filtered out any site with a mean coverage greater than twice the genome-wide median as likely caused by duplication [21]. This removes variants found in repetitive regions, such as centromeric sequence. We also filtered out any site with less than $2 \times$ coverage per person in the study population as being too poorly covered to accurately call SNPs. Finally, we removed sites that had an excess of heterozygotes compared to expectations from Hardy-Weinberg equilibrium. We tested this using a test from Emigh [44] describing the heterozygote probability as:

$$
P_{A a}=\frac{n !}{n_{A A} ! n_{A a} ! n_{a a} !} * \frac{n_{A} ! n_{a} !}{(2 n) !} * 2^{n_{A a}}
$$

Variants with $P$-value $<0.05$ were discarded. After filtering variants based on the three filters described above, we remained with 7,555,907 SNPs in the Amhara population and 8,069,425 SNPs in the Oromos population. See Additional file 17 for an exemplar of the number of variants removed in each filtering step. In addition, to exclude cryptic relatedness in the Oromos and Amhara population samples, we applied PLINK's [45] $\hat{\pi}$ test for identity by descent. This resulted in excluding four individuals from our initial Oromos sample, finally arriving at six Oromos and seven Amhara individuals.

\section{Lowlander control populations}

To identify appropriate controls, we used low coverage whole-genome sequencing calls from the 1000 Genomes Project populations [21]. We ran ADMIXTURE [20] on 13,928 sampled sites to identify the population most closely related to the highlanders. As seen in Additional file 2, the Ethiopian individuals consist largely of African ancestry, but possess a more substantial European component compared to the other African populations. The closest population consists of 67 Luhya (LWK) individuals from Webuye, Kenya, and was thus chosen as the control for all cross-population tests of selection (see below). As an out-group for the PBS test (see below), we used 90 European (CEU) individuals in order to capture variation in the highlanders shared with individuals of European ancestry. We also performed principle component analysis on our study populations jointly with the lowlander controls (LWK) and out-group (CEU), further illustrating our study populations as an admixture of these two ancestral groups (Additional file 3).

\section{Identifying regions under positive selection}

Under positive selection, haplotypes carrying the beneficial mutation (as well as linked, neutral mutations) rapidly increase in frequency, leading to a loss of genetic diversity in the region surrounding the mutation [46] (illustration in Additional file 5). This loss of diversity, or selective sweep, decreases with distance from the beneficial mutation due to recombination. The loss of allelic diversity and the corresponding skew in the allele frequency spectrum can be used to detect loci important for adaptation to the selective stress [46]. We use crosspopulation tests to adjust for interesting frequency profiles that are shared between our study and control populations. These are likely due to events (such as bottlenecks, genetic drift, or even selection for a different phenotype) occurring before our study and control populations diverged, and thus likely not related to hypoxia tolerance. Population-specific selection can be measured by comparing the estimated scaled mutation rate $\theta=4 N_{\mathrm{e}} \mu$ in a given loci to that of the same loci in a control population. A large decrease in $\theta$ of the study population compared to controls indicates a region is evolving non-neutrally in the study population, consistent with positive selection. It is important to note that tests of selection may be confounded by several factors, including demographic events (for example, severe bottlenecks) and genetic drift [23]. We therefore use experimental validation in a model system as independent validation that our tests identified adaptive regions.

\section{Tests of selection}

First, we ran two cross-population tests comparing the Amhara or Oromos populations (study) against the 1000 Genomes Luhya population (control). These tests are based on two common estimators of $\theta$ : the summed non-fixed frequencies estimator, denoted $\theta_{f}$, and the average pairwise heterozygosity estimator, denoted $\theta_{\pi}$ [47]. For a given region, a high $\log$ ratio of $\theta_{\pi}\left(\theta_{\mathrm{f}}\right)$ in the control relative to the study population is indicative of selection [48]. We label these log ratio statistics as $S_{\text {п }}$ for the average heterozygosity estimator and $S_{\mathrm{f}}$ for the summed frequency estimator, such that:

$$
\begin{gathered}
S_{f}=\log \left(\frac{\theta_{f, \text { control }}}{\theta_{f, \text { study }}}\right) \\
S_{\pi}=\log \left(\frac{\theta_{\pi, \text { control }}}{\theta_{\pi, \text { study }}}\right)
\end{gathered}
$$

Another class of tests for selection is based on the fixation index, or $F_{s t}$, between two populations [49]. This class aggregates differential SNP frequencies across two populations. For instance, Hudson et al. [50] define this measure as:

$$
F_{s t}=1-\frac{\pi_{w}}{\pi_{b}}
$$

Where $\pi_{\mathrm{w}}$ represents the within-population average heterozygosity and $\pi_{\mathrm{b}}$ represents the between-population 
average heterozygosity. As two populations diverge, the variability between the populations increases much more than the variability within each population, and the statistic approaches one. The fixation index roughly correlates to the evolutionary branch length $T$ between two populations [51] as:

$$
T=-\log \left(1-F_{s t}\right)
$$

This approach is not directional, however. As a result, a significant statistic value may indicate a selective sweep in either the study or the control population. To address this, Shriver et al. [52] and Yi et al. [10] developed the concept of a population branch statistic, or PBS. This combines the pairwise branch lengths of three populations as follows:

$$
P B S=\frac{T^{S N}+T^{S O}-T^{N O}}{2}
$$

Where $\mathrm{S}$ represents a study population, $\mathrm{N}$ represents an evolutionarily close control population, and $\mathrm{O}$ represents a distant out-group. We calculated the PBS with our study population defined as either the Amhara or Oromos population, our control as the Luhya population, and our out-group as the CEU population. Additionally, we compared the results of the above tests with XP-CLR [53], a method that attempts to detect large linkage blocks with high frequency differential as indicative of positive selection.

For $\mathrm{S}_{\mathrm{f}}, \mathrm{S}_{\pi}, \mathrm{F}_{\mathrm{st}}$, and PBS, we use genomic windows of size $50 \mathrm{kbp}$, overlapping at $2 \mathrm{kbp}$ intervals. For each test, we define the top $0.1 \%$ genome-wide value as the genomic-control cutoff to determine the windows of interest. The code used to compute these test statistics can be downloaded from [54].

For the XP-CLR test statistic, we found that using a $0.1 \%$ genome-wide threshold was overly stringent. Testing Amhara versus Luhya using a 0.1\% threshold, exactly five non-overlapping regions exceeded the threshold, all of which contained highly repetitive sequence (except for the HLA region, which has a high mutation rate). Relaxing the threshold to $0.3 \%$ genome-wide yielded a comparable number of regions to that found by our other tests, but since XP-CLR uses variable size genomic windows (normally much larger than $50 \mathrm{kbp}$ ), the list of implicated genes was dominated by XP-CLR results. Hence, we used XP-CLR only for secondary validation. For instance, the EDNRB gene region on chromosome 13 was found to be significant using XP-CLR under a $0.3 \%$ threshold.

\section{Population simulations and power estimation}

We generated simulated populations using the mpop forward simulator [55] and Hudson's ms coalescent simulator [56]. For a given set of parameters $\mu, r, s, t$ (mutation rate, recombination rate, selection coefficient and time since selection, respectively) we generated 200 sets of simulated populations. We initiated each instance with a unique source population of $\mathrm{N}_{\mathrm{e}}=1,000$ diploids from a neutral coalescent process, using Hudson's $m s$ simulator. We then sampled with replacement from the source population into three separate populations of size $N_{e}$ each, labeled 'study', 'cont1', and 'cont2'. We evolved these populations separately using the mpop simulator, such that only the study population had a locus under positive selection. Individuals carrying the advantageous allele had higher likelihood ( $\propto 1+s$, for a homozygous carrier) to reproduce at each generation. The other populations (cont1 and cont2) continued to evolve neutrally. After $\tau$ generations, a random sample ( $n=100$ diploids) was taken from each of the three populations, and crosspopulation neutrality tests were applied. Genomic regions of size $50 \mathrm{kbp}$ were simulated, with mutation and recombination rates set to $\mu=2.4 \times 10^{-7}$ and $r=3.784 \times 10^{-8}$ per base, per generation. The selection coefficient used for these simulations was $s=0.02$, and the number of generations since selection $\tau$ ranged between [50, 4000].

The power of a test statistic at $5 \%$ false positive rate was determined as the fraction of statistic values exceeding a certain cutoff when applied to the study versus cont1 samples. Cutoff values were set to the top $5 \%$ of the null distribution, obtained by applying the same test to samples from two simulated populations evolving neutrally (cont1 versus cont2).

Power of different tests under varying model parameters

The different tests for selection described above all aim to find regions with marked differences in allele frequencies across study and control populations. However, the specific signal observed is highly influenced by different factors, such as the selection coefficient and the time since selection. In general, the allelic divergence in a region is a function of the local mutation and recombination rates. Under a Wright-Fisher model of neutral evolution, the expected distribution of allele frequencies (the site frequency spectrum) is known. Specifically, the expected number of alleles with frequency $f$, where $f=$ $(1, \ldots, n-1)$, is given by $\theta / f[47]$. Under selective pressure, the site frequency spectrum begins to shift [57]. Initially, the haplotypes carrying the beneficial alleles rapidly increase in frequency, reducing the overall divergence (a selective sweep). Shortly after the beneficial allele becomes fixed in the population, the divergence is at its lowest, and the signal of selection is strongest. As time passes, de novo mutations and recombination events gradually restore variability to the region. Initially, there is an increase in low frequency alleles, which then reach intermediate and high frequencies, finally drowning out 
the selection signal. Thus, there are three major regimes for a population under positive selection: 'pre-fixation', where the beneficial haplotype starts to rise in frequency; 'near fixation', where the haplotype approaches fixation; and 'post-fixation', where the haplotype is fixed in the population, and de novo mutations slowly restore diversity to the population. In these regimes, the four tests show different relative strengths in detecting positive selection (Additional file 4). Importantly, although in the example shown the selection coefficient was set to $s=0.02$, the performance of the different tests diverges further under different selection pressures (where some tests dominate in weaker selection and others in stronger selection) as well as under different demographic histories [22].

\section{$S_{f}$ test}

The $S_{\mathrm{f}}$ test sums non-fixed frequencies in a region, effectively down-weighting low frequency alleles [58]. The result of this is that the $S_{\mathrm{f}}$ test is optimized for the postfixation regime, since in this regime, the bulk of the signal comes from the reduced number of intermediate and high frequency alleles in the study population. As de novo mutations only approach these frequencies many generations after fixation, $S_{f}$ is able to detect selection for longer periods of time after fixation has occurred than other tests. In addition, $\mathrm{S}_{\mathrm{f}}$ excels at capturing stronger selection pressures, where the beneficial allele goes to fixation relatively quickly and thus reaches the postfixation regime sooner. As Additional file 4 shows, once in the post-fixation regime, the peak power for $S_{\mathrm{f}}$ is sustained for more generations compared to other tests.

\section{$S_{\pi}$ test}

The $S_{\pi}$ test is similar to $S_{\mathrm{f}}$ in that it is sensitive to strong selection pressures, as well as long times since selection start. However, $S_{\pi}$ is based on average heterozygosity, which weights allelic differences identically (in other words, $S_{\pi}$ returns the same value if the derived allele is defined as either the major or the minor allele). This essentially folds the frequency spectrum [58], leading to two major benefits. First, an approach such as $S_{\mathrm{f}}$ depends heavily on the idea that the ancestral allele is the reference nucleotide. If this is not true, for instance, a variant at $10 \%$ frequency can be mistaken for a variant at $90 \%$ frequency instead, heavily impacting the statistic value. For a folded spectrum, however, this is not the case. In addition, unlike $S_{f}, S_{\pi}$ can detect the loss of diversity due to a loss of intermediate frequency alleles, causing it to pick up selection prior to fixation (where there is an abundance of high frequency alleles). However, since it folds the spectrum, $\mathrm{S}_{\pi}$ cannot distinguish high frequency from low frequency variants, and thus only has high power until de novo mutations reach intermediate frequencies.

\section{$F_{\text {st }}$ and PBS tests}

As mentioned previously, under positive selection, as the beneficial haplotype dominates the study population, the variability within this population decreases. This can be tested directly using the relative allele frequency spectra as in $S_{\mathrm{f}}$ and $\mathrm{S}_{\pi}$, but there is additional information in the site-specific frequency differences across the study and control populations. For instance, let us consider a variant at $20 \%$ frequency in the control population. In the study population, this variant lies on a beneficial haplotype, and is sampled at frequency $80 \%$. Under $S_{\pi}$, this variant contributes equally to both the study and the control statistics, while in $\mathrm{S}_{\mathrm{f}}$, this variant contributes negatively to the overall statistic. However, there is clearly a sharp rise in frequency, representing an increased branch length between the study and control populations in the phylogenetic tree, which may be indicative of selection. Since the $F_{\text {st }}$ test measures the sitespecific variability between populations $\left(\pi_{\mathrm{b}}\right)$, it would be able to detect such situations. Importantly, the scenario described above is consistent with selection occurring on standing variation, where the beneficial haplotype is present in non-negligible frequencies in the control population. However, the undirected nature of the branch lengths presents disadvantages. For instance, a significant $\mathrm{F}_{\mathrm{st}}$ value could also indicate positive selection in the control population. This is addressed in the PBS test by calculating population-specific branch lengths using multiple controls.

\section{Effects of sample size on power}

In our study, we performed high coverage (15 to 20x), WGS on seven Amhara and six Oromos individuals. Alternative approaches to WGS would include exome sequencing or genotyping. As these approaches are currently less expensive, this may allow for sampling more individuals. In Additional file 15, we show the impact of sample size on power, using simulated populations. The simulation procedure was similar to the one described above, with 500 neutral initial populations, and selection coefficient fixed at $s=0.02$. As previously described (Additional file 5), there appear to be three general regimes of selection ('pre-fixation', 'near fixation', and 'post-fixation'), where different tests vary in their relative performance across regimes. We focus on a single test, $S_{\pi}$, and sample from each of the three regimes ( $\tau=450,700,1,000,1,500$ generations after selection begins). In order to identify the effect of decreasing the sample size on power, we vary the sample sizes from $n=$ $(2, \ldots, 40)$. As a gold standard for maximal attainable power, we used a large sample size of $n=400$. Although sequencing more individuals would improve the sensitivity, as seen in Additional file 15, sampling 12 or 14 haplotypes yields between 67 and 95\% power compared to 
our gold standard. Notably, we see that sampling fewer individuals has the greatest impact in the 'pre-fixation' regime. This is due to two factors. First, sampling fewer individuals leads to higher variance in the observed frequencies (Additional file 8). Second, the pre-fixation regime is when the frequency differential of the beneficial haplotype block compared to controls is lowest. Despite this, we are still able to detect positive selection in the majority of our simulated cases.

\section{Comparison of whole genome sequencing with other assays}

We also tested the power of whole genome sequencing in comparison to other technologies, due to its unique ability to capture all allelic variation in a region. Additional file 14 shows the $S_{\pi}$ test applied to chromosome 13, which contains one of our top hits (the EDNRB gene region). We compared the variants captured in our study to those captured by two alternative approaches: whole exome sequencing and genotyping. To mimic the effects of whole exome sequencing, we masked variants not targeted by the Nimblegen 2.1 M exon capture array. For comparison with genotyping studies, we masked variants not included in the approximately $1 \mathrm{M}$ Affymetrix Genome-Wide Human SNP Array 6.0. As shown in Additional file 14, with WGS the strongest signal chromosome-wide is located in the EDNRB gene region. In contrast, genotyping shows a significantly weaker signal in the region, while whole exome sequencing shows no signal at all. Thus, for situations where a large portion of the signal is in intergenic or intronic sequence, WGS may provide a major advantage over other technologies.

\section{Drosophila stocks and test of hypoxia tolerance}

The D. melanogaster stocks carrying UAS-RNAi transgene were obtained from the Vienna Drosophila RNAi Center (Vienna, Austria; stock numbers 25995 and 103805 carrying UAS-RNAi(cic); 22358 and 109336 carrying UAS-RNAi $(H s l) ; 29003$ and 107333 carrying UASRNAi $(P a f-A h \alpha) ; 42462$ and 8018 carrying UAS-RNAi (CG7466)). The da-Gal4 driver (stock number 8641) was obtained from Bloomington stock center (Bloomington, IN, USA).

Hypoxia tolerance of Drosophila crosses with specific RNAi-mediated knockdown was carried out as described in [16]. Fifteen virgin female flies homozygous for UASRNAi were crossed with 10 male flies homozygous for da-GAL4 and allowed to lay eggs for 24 hours in normoxia. The vials with the eggs were transferred into a computer-controlled atmosphere chamber supplied with $5 \%$ oxygen balanced with nitrogen, with 12 hour-dark and 12 hour-light cycle at $22 \pm 1^{\circ} \mathrm{C}$. The Gal4 driver and UAS-RNAi stocks alone without crossing were included in parallel as controls. After three weeks of culturing, the vials were assayed for the number of pupal cases that were empty or full to calculate the eclosion rate. Six vials of each condition were completed in 2 different experiments for a minimum of 200 pupal cases scored for each condition/cross. The eclosion rate was presented as percentage of empty pupae in all scored pupal cases.

\section{Data availability}

Genotype data have been deposited in dbGaP under accession number phs000647.v1.p1.

\section{Additional files}

Additional file 1: Figure S1. Computational analysis workflow. The raw reads were mapped using BWA, followed by indel realignment, duplicate marking, and quality score recalibration using the GATK pipeline. Variants were then called and filtered using GATK's UnifiedGenotyper. After applying additional variant filters to account for the differences in coverage between the study and control populations, we applied several complementary tests to identify 420 regions as candidates for positive selection. Of these, 412 were filtered using 4 prioritization filters customized to the challenges of our sequencing framework, leading to 8 final prioritized regions.

Additional file 2: Figure S2. ADMIXTURE analysis with six clusters on the Ethiopian highlanders, along with the 1000 Genomes populations. The highlander ancestry is a mixture of traditionally African and traditionally European genotypes, represented by the green and dark blue segments, respectively. Within the African 1000 Genomes populations, the nearest population geographically as well as ancestrally appears to be the Luhya (LWK) population. We thus selected this population as our control. Similarly, the section sharing ancestry with European populations appears closer to the southern and western Europeans than the Finnish population. As a result, as outgroup in the PBS test, we used the CEU population.

Additional file 3: Figure S3. Principal component analysis of the Ethiopian highlanders, along with the 1000 Genomes control (LWK) and out-group (CEU). As can be seen, the first principal component separates the four population samples, further illustrating that the Amhara and Oromos highlanders posses a mixture of African and European ancestry.

Additional file 4: Figure S4. Power of neutrality tests used in this study $\left(S_{\pi}, F_{s t}, S_{f}\right.$ and PBS) as function of time. (A) The $x$-axis scales linearly in terms of generations since selection start. (B) Power as function of logarithmically scaled time for the neutrality tests used in this study. We also show the $x$-axis in units of $\ln (2 \mathrm{Ns}) / \mathrm{s}$ (top axis), which can define the regimes as a function of selection pressure. We observe three major regimes, corresponding to the state of the beneficial haplotype in the case population: before the haplotype has significantly risen in frequency ('pre-fixation'), as the haplotype dominates the case population ('near fixation'), and after the haplotype has gone to fixation, while the frequency spectrum gradually reverts to neutrality ('post-fixation'). In these three regimes, the statistics perform differently: PBS performs better in the first regime, $S_{\pi}$ performs best in the second regime, and $S_{f}$ dominates the third regime.

Additional file 5: Figure S5. Illustration of a selective bottleneck in one of two diverged populations, leading to a loss of genetic diversity. The haplotype carrying the beneficial allele (shown in blue) becomes dominant in the population under selection, at the expense of other haplotypes that die out (black lines near the selective bottleneck). This leads to decreased genetic diversity, characterized by a skew in the site frequency spectrum (top) relative to neutrality (bottom). As time progresses, genetic diversity is gradually restored to the region via de novo mutation (seen in the 'post-fixation' regime).

Additional file 6: Table S1. Regions identified as significant under a $0.1 \%$ genome-wide false discovery rate. 
Additional file 7: Supplementary Dataset 1. Regions exceeding the $1 \%$ genome-wide FDR for the four tests of selection $\left(S_{\pi}, S_{f}, F_{s t}\right.$ and PBS) and the final list of prioritized regions. In addition, it contains a list of nonsynonymous SNPs within the final regions, as well as SNPs in ENCODE and TRANSFAC transcription factor binding sites overlapping these regions. Provided as a separate Excel file with the tables as sheets. The file can be viewed with Microsoft Excel Viewer.

Additional file 8: Figure S6. The impact of sampling haplotypes from a population on observed allele frequencies. The red (blue) line shows the $95 \%$ confidence interval (Cl) of observed frequency when sampling $n=12(n=14)$ haplotypes from a population. This corresponds to our Oromos and Amhara population samples, respectively. For most intermediate frequencies, a difference of around $20 \%$ is within the $95 \%$ $\mathrm{Cl}$. We use the $95 \% \mathrm{Cl}$ frequency difference as a cutoff, prioritizing regions containing haplotype blocks with a greater frequency differential between the highlander population and lowlander controls. For regions on the $\mathrm{X}$ chromosome, the number of sampled haplotypes is half, and we therefore required a greater frequency differential (approximately $40 \%$ for intermediate frequencies).

Additional file 9: Test statistic values on chromosomes 1 to 22 , as well as $\mathrm{X}$, in the Amhara and the Oromos populations. The tests shown are $\mathrm{PBS}, \mathrm{F}_{\mathrm{St}}, \mathrm{S}_{\pi}$, and $\mathrm{S}_{\mathrm{f}}$. Regions exceeding the $0.1 \%$ genomic FDR and that passed all prioritization criteria are shown in green.

Additional file 10: Figure S7. Linkage disequilibrium (LD) near the chromosome 19 region. In Oromos (top left), Amhara (top right), as well as the two 1000 Genomes lowlander controls: LWK (bottom left) and CEU (bottom right). The center of the region is marked by a black star. We observe a strong, and large, LD block surrounding the chromosome 19 region in Oromos. A corresponding, but smaller, block is also visible in the Amhara. This observation is in line with the longer time spent at high altitude by the Amhara population, during which recombination may have broken local LD structure. We note that the overall higher levels of LD observed in the Oromos and Amhara may be due to smaller sample sizes in these populations, but that this should be mostly a background effect, and is thus not expected to significantly alter the observed block structure.

Additional file 11: Table S2. Nonsynonymous SNPs with significant frequency differential in one of the eight prioritized regions.

Additional file 12: Figure S8. Evidence supporting EDNRB as a gene candidate. Top panel: $S_{\pi}$ statistic values across chromosome 13 in the Amhara population, compared to the Luhya (LWK) population. The red line represents a genome-wide, 0.1\% FDR. Two distinct regions exceed this cutoff, one of which did not show a haplotype block with significant frequency differential compared to our lowlander controls, and was thus removed from consideration. Bottom panel: SNP frequency profile of the significant region in the Amhara (blue) compared to Luhya (brown, inverted) populations. As can be seen, variant frequencies in this region are much higher in the Amhara population compared to lowlander controls.

Additional file 13: Table S3. Clinical characteristics of Oromos and Amhara subjects.

Additional file 14: Figure S9. Impact of whole genome sequencing on selection signals. (A-C) $S_{\pi}$ statistic values across chromosome 13 in the Amhara population compared to the Luhya (LWK) population, using the complete set of variants from whole genome sequencing (A), the subset that overlap targets from whole exome capture (B), and the subset (about $1 \mathrm{M}$ ) that overlaps a popular genotyping array (C). The red lines represent the respective genome-wide $0.1 \%$ FDR calculated individually for each case. Highlighted in green is the EDNRB gene loci. (D-F) SNP frequency profiles of the EDNRB region in Amhara (blue) compared to Luhya (brown, inverted) for whole genome sequencing (D), whole exome sequencing $(E)$, and genotyping $(F)$. As can be seen from the green highlighted regions $(\mathrm{A}-\mathrm{C})$, the strong signal present when considering whole genome sequencing is reduced drastically with genotyping and is entirely absent with exome sequencing.

Additional file 15: Figure S10. The impact of sequenced sample size on power, using $\mathrm{S}_{\pi}$ as an exemplar test. Five hundred populations were simulated with a fixed selection coefficient of $s=0.02$ and sampled at different times after selection start. Sample size is shown in haplotypes, and ranges in $n=(2,3, \ldots, 40)$. Optimal power at each time was determined using a large sample size of $n=400$. The populations were sampled at four time points representing each of the observed regimes: $t=450$ for 'pre-fixation', $t=700$ and $t=1,000$ for 'near-fixation', and $t=1,500$ for 'post-fixation'. Although we see an increase in power as more haploptypes are sampled, sampling 12 or 14 haplotypes (our Oromos and Amhara populations, respectively) yields 67 to $95 \%$ of the optimal power.

Additional file 16: Table S4. Sequencing depth and coverage statistics per individual in the sample.

Additional file 17: Table S5. Number of variants removed in each filtering step, for the Luhya and Oromos populations.

\section{Abbreviations}

bp: base pair; Cl: confidence interval; FDR: false discovery rate; HIF: hypoxia inducible factor; LD: linkage disequilibrium; PBS: population branch statistic; RNAi: RNA interference; SNP: single nucleotide polymorphism; WGS: whole genome sequencing.

\section{Competing interests}

The authors declare that they have no competing interests.

\section{Authors' contributions}

$D Z, V B$, and $G G H$ designed the experiments with input from $N U, R R$, JX, and KAF. NU, RR, and VB performed sequence and variation analysis, including tests for positive selection. JL, YY, YD, LG, RC, YW, XJ, CH, WJ, DC, GG, SL, and $Y L$ performed whole genome sequencing and read alignment. TS, JX, and DZ performed hypoxia tolerance analysis in Drosophila. OA, VEC, RH, JLG, MZ, and $\mathrm{GZ}$ collected human blood samples. NU, RR, DZ, VB, and GGH wrote the manuscript with comments from co-authors. NU, RR, DZ, VB, and GGH contributed equally to the project. All authors read and approved the final manuscript.

\section{Acknowledgments}

We thank Yu-hsin Hsiao, Orit Poulsen, Efrat Golan, and Shewadeg Gebru for technical assistance. We also thank Dr Cynthia Beall and Dr Anna Di Rienzo for providing Ethiopian highlander genotype data. This study was supported by NSF-CCF-1115206, NSF-IIS-1318386, 5RO1-HG004962 and U54 HL108460 to VB, and NIH awards 1P01HL098053 and 5P01HD32573 to GGH.

\section{Author details}

${ }^{1}$ Bioinformatics \& Systems Biology Graduate Program, University of California San Diego, La Jolla, California 92093, USA. ${ }^{2}$ Department of Pediatrics, Division of Respiratory Medicine, University of California San Diego, La Jolla, California 92093, USA. ${ }^{3}$ BGI-Americas, Cambridge, Massachusetts 02142, USA.

${ }^{4}$ Department of Neurology, New Mexico Health Enhancement and Marathon Clinics Research Foundation, Albuquerque, New Mexico 87122, USA. ${ }^{5} \mathrm{BGl}$-Shenzhen, Shenzhen 518083, China. ${ }^{6}$ Department of Biomedical Physiology and Kinesiology, Faculty of Science, Simon Fraser University, British Columbia, Canada V5A 16S. ${ }^{7}$ Division of Cardiovascular and Neuronal Remodeling, Faculty of Medicine, University of Leeds, Leeds LS2 9JT, UK. ${ }^{8}$ Division of Clinical Pharmacology/Department of Medicine, Vanderbilt University Medical Center, Tennessee 37232, USA. ${ }^{9}$ Department of Medicine, Yehuleshet Higher clinic, University of Addis Ababa, Addis Ababa 1176, Ethiopia. ${ }^{10}$ Beijing Institute of Genomics, Chinese Academy of Sciences, Beijing 100029, China. " Department of Pediatrics, Division of Genome Information Science, University of California San Diego, La Jolla, California 92093, USA. ${ }^{12}$ Department of Computer Science and Engineering, University of California San Diego, La Jolla, California 92093, USA. ${ }^{13}$ Department of Neurosciences, University of California San Diego, La Jolla, California 92093, USA. ${ }^{14}$ Rady Children's Hospital, San Diego, California 92123, USA.

Received: 9 November 2013 Accepted: 20 February 2014 Published: 20 February 2014

\section{References}

1. Rupert JL, Hochachka PW: The evidence for hereditary factors contributing to high altitude adaptation in Andean natives: a review. High Alt Med Biol 2001, 2:235-256. 
2. Moore LG, Niermeyer S, Zamudio S: Human adaptation to high altitude: regional and life-cycle perspectives. Am J Phys Anthropol 1998, 27:25-64. 3. Beall CM: Two routes to functional adaptation: Tibetan and Andean high-altitude natives. Proc Natl Acad Sci USA 2007, 104:8655-8660.

4. Beall CM, Decker MJ, Brittenham GM, Kushner I, Gebremedhin A, Strohl KP: An Ethiopian pattern of human adaptation to high-altitude hypoxia. Proc Natl Acad Sci U S A 2002, 99:17215-17218.

5. Appenzeller O, Claydon VE, Gulli G, Qualls C, Slessarev M, Zenebe G, Gebremedhin A, Hainsworth R: Cerebral vasodilatation to exogenous NO is a measure of fitness for life at altitude. Stroke 2006, 37:1754-1758.

6. Beall CM: High-altitude adaptations. Lancet 2003, 362:14-15.

7. Beall CM: Detecting natural selection in high-altitude human populations. Respir Physiol Neurobiol 2007, 158:161-171.

8. Pagani L, Ayub Q, MacArthur DG, Xue Y, Baillie JK, Chen Y, Kozarewa I, Turner DJ, Tofanelli S, Bulayeva K, Kidd K, Paoli G, Tyler-Smith C: High altitude adaptation in Daghestani populations from the Caucasus. Hum Genet 2012, 131:423-433.

9. Moore LG, Shriver M, Bemis L, Hickler B, Wilson M, Brutsaert T, Parra E, Vargas E: Maternal adaptation to high-altitude pregnancy: an experiment of nature - a review. Placenta 2004, 25:60-71.

10. Yi X, Liang Y, Huerta-Sanchez E, Jin X, Cuo ZXP, Pool JE, Xu X, Jiang H, Vinckenbosch N, Korneliussen TS, Zheng H, Liu T, He W, Li K, Luo R, Nie X, Wu H, Zhao M, Cao H, Zou J, Shan Y, Li S, Yang Q, Asan, Ni P, Tian G, Xu J, Liu $X$, Jiang T, Wu R, et al: Sequencing of 50 human exomes reveals adaptation to high altitude. Science 2010, 329:75-78.

11. Simonson TS, Yang Y, Huff CD, Yun H, Qin G, Witherspoon DJ, Bai Z, Lorenzo FR, Xing J, Jorde LB, Prchal JT, Ge R: Genetic evidence for high-altitude adaptation in Tibet. Science 2010, 329:72-75.

12. Beall CM, Cavalleri GL, Deng L, Elston RC, Gao Y, Knight J, Li C, Li JC, Liang Y, McCormack M, Montgomery HE, Pan H, Robbins PA, Shianna KV, Tam SC, Tsering N, Veeramah KR, Wang W, Wangdui P, Weale ME, Xu Y, Xu Z, Yang L, Zaman MJ, Zeng C, Zhang L, Zhang X, Zhaxi P, Zheng YT: Natural selection on EPAS1 (HIF2alpha) associated with low hemoglobin concentration in Tibetan highlanders. Proc Natl Acad Sci U S A 2010, 107:11459-11464.

13. Scheinfeldt LB, Soi S, Thompson S, Ranciaro A, Woldemeskel D, Beggs W, Lambert C, Jarvis JP, Abate D, Belay G, Tishkoff SA: Genetic adaptation to high altitude in the Ethiopian highlands. Genome Biol 2012, 13:R1.

14. Alkorta-Aranburu G, Beall CM, Witonsky DB, Gebremedhin A, Pritchard JK, Di Rienzo A: The genetic architecture of adaptations to high altitude in Ethiopia. PLoS Genet 2012, 8:e1003110.

15. Huerta-Sánchez E, DeGiorgio M, Pagani L, Tarekegn A, Ekong R, Antao T, Cardona A, Montgomery HE, Cavalleri GL, Robbins PA, Weale ME, Bradman N, Bekele E, Kivisild T, Tyler-Smith C, Nielsen R: Genetic signatures reveal high-altitude adaptation in a set of Ethiopian populations. Mol Biol Evol 2013, 30:1877-1888.

16. Zhou D, Udpa N, Gersten M, Visk DW, Bashir A, Xue J, Frazer KA, Posakony JW, Subramaniam S, Bafna V, Haddad GG: Experimental selection of hypoxia-tolerant Drosophila melanogaster. Proc Natl Acad Sci U S A 2011, 108:2349-2354.

17. Li H, Durbin R: Fast and accurate short read alignment with BurrowsWheeler transform. Bioinformatics 2009, 25:1754-1760.

18. McKenna A, Hanna M, Banks E, Sivachenko A, Cibulskis K, Kernytsky A, Garimella K, Altshuler D, Gabriel S, Daly M, DePristo MA: The Genome Analysis Toolkit: a MapReduce framework for analyzing next-generation DNA sequencing data. Genome Res 2010, 20:1297-1303.

19. DePristo MA, Banks E, Poplin R, Garimella KV, Maguire JR, Hartl C, Philippakis AA, del Angel G, Rivas MA, Hanna M, McKenna A, Fennell TJ, Kernytsky AM, Sivachenko AY, Cibulskis K, Gabriel SB, Alshuler D, Daly MJ: A framework for variation discovery and genotyping using next-generation DNA sequencing data. Nat Genet 2011, 43:491-498.

20. Alexander DH, Novembre J, Lange K: Fast model-based estimation of ancestry in unrelated individuals. Genome Res 2009, 19:1655-1664.

21. The 1000 Genomes Project Consortium: A map of human genome variation from population-scale sequencing. Nature 2010, 467:1061-1073.

22. Ronen R, Udpa N, Halperin E, Bafna V: Learning natural selection from the site frequency spectrum. Genetics 2013, 195:181-193.

23. Teshima KM, Coop G, Przeworski M: How reliable are empirical genomic scans for selective sweeps? Genome Res 2006, 16:702-712.

24. Akey JM: Constructing genomic maps of positive selection in humans: where do we go from here? Genome Res 2009, 19:711-722.
25. The International HapMap Consortium: A second generation human haplotype map of over 3.1 million SNPs. Nature 2007, 449:851-861.

26. Marcus HG: A History of Ethiopia. Updated edition. Berkeley and Los Angeles, CA: University of California Press; 2002.

27. Bernard N, Girouard J, Forest JC, Giguere Y: The combination of ApoCIII, hepatic lipase and hormono sensitive lipase gene polymorphisms suggests an association with susceptibility to gestational hypertension. J Hum Genet 2007, 52:244-254.

28. Lampidonis AD, Rogdakis E, Voutsinas GE, Stravopodis DJ: The resurgence of Hormone-Sensitive Lipase (HSL) in mammalian lipolysis. Gene 2011, 477:1-11.

29. Weber GJ, Choe SE, Dooley KA, Paffett-Lugassy NN, Zhou Y, Zon LI: Mutantspecific gene programs in the zebrafish. Blood 2005, 106:521-530.

30. Iqbal J, Weisenburger DD, Chowdhury A, Tsai MY, Srivastava G, Greiner TC, Kucuk C, Deffenbacher K, Vose J, Smith L, Au WY, Nakamura S, Seto M, Delabie J, Berger F, Loong F, Ko Y-H, Sng I, Liu X, Loughran TP, Armitage J, Chan WC: Natural killer cell lymphoma shares strikingly similar molecular features with a group of non-hepatosplenic gamma-delta T-cell lymphoma and is highly sensitive to a novel aurora kinase $\mathrm{A}$ inhibitor in vitro. Leukemia 2011, 25:348-358.

31. Chen $\mathrm{CH}$ : Platelet-activating factor acetylhydrolase: is it good or bad for you? Curr Opin Lipidol 2004, 15:337-341.

32. Arai $\mathrm{H}$, Koizumi $\mathrm{H}$, Aoki J, Inoue K: Platelet-activating factor acetylhydrolase (PAF-AH). J Biochem 2002, 131:635-640.

33. Clozel M, Gray GA, Breu V, Loffler BM, Osterwalder R: The endothelin ETB receptor mediates both vasodilation and vasoconstriction in vivo. Biochem Biophys Res Commun 1992, 186:867-873.

34. McCulloch KM, MacLean MR: EndothelinB receptor-mediated contraction of human and rat pulmonary resistance arteries and the effect of pulmonary hypertension on endothelin responses in the rat. J Cardiovasc Pharmacol 1995, 26:S169-S176.

35. Pritchard JK, Pickrell JK, Coop G: The genetics of human adaptation: hard sweeps, soft sweeps, and polygenic adaptation. Curr Biol 2010, 20:R208-R215.

36. Azad P, Zhou D, Zarndt R, Haddad GG: Identification of genes underlying hypoxia tolerance in Drosophila by a P-element screen. G3 (Bethesda) 2012, 2:1169-1178.

37. Astigarraga S, Grossman R, Diaz-Delfin J, Caelles C, Paroush Z, Jimenez G: A MAPK docking site is critical for downregulation of Capicua by Torso and EGFR RTK signaling. EMBO J 2007, 26:668-677.

38. Tseng AS, Tapon N, Kanda H, Cigizoglu S, Edelmann L, Pellock B, White K, Hariharan IK: Capicua regulates cell proliferation downstream of the receptor tyrosine kinase/ras signaling pathway. Curr Biol 2007, 17:728-733.

39. Hurlbut GD, Kankel MW, Artavanis-Tsakonas S: Nodal points and complexity of Notch-Ras signal integration. Proc Natl Acad Sci U S A 2009, 106:2218-2223.

40. Bigham AW, Mao X, Mei R, Brutsaert T, Wilson MJ, Julian CG, Parra EJ, Akey JM, Moore LG, Shriver MD: Identifying positive selection candidate loci for highaltitude adaptation in Andean populations. Hum Genomics 2009, 4:79-90.

41. Sabeti PC, David ER, John MH, Haninah ZPL, Daniel JR, Stephen FS, Stacey BG, Platko JV, Patterson NJ, McDonald GJ, Ackerman HC, Campbell SJ, Altshuler D, Cooper R, Kwiatkowski D, Ward R, Lander ES: Detecting recent positive selection in the human genome from haplotype structure. Nature 2002, 419:832-837.

42. Leon-Velarde F, McCullough RG, McCullough RE, Reeves JT: Proposal for scoring severity in chronic mountain sickness (CMS). Adv Exp Med Biol 2003, 543:339-354

43. Li R, Li Y, Fang X, Yang H, Wang J, Kristiansen K, Wang J: SNP detection for massively parallel whole-genome resequencing. Genome Res 2009, 19:1124-1132.

44. Emigh $\mathrm{TH}$ : A comparison of tests for Hardy-Weinberg equilibrium. Biometrics 1980, 36:627-642.

45. Purcell S, Neale B, Todd-Brown K, Thomas L, Ferreira MAR, Bender D, Maller J, Sklar P, de Bakker PIW, Daly MJ, Sham PC: PLINK: a tool set for whole-genome association and population-based linkage analyses. Am J Hum Genet 2007, 81:559-575.

46. Sabeti PC, Schaffner SF, Fry B, Lohmueller J, Varilly P, Shamovsky O, Palma A, Mikkelsen TS, Altshuler D, Lander ES: Positive natural selection in the human lineage. Science 2006, 312:1614-1620.

47. Fu YX: Statistical properties of segregating sites. Theor Popul Biol 1995, 48:172-197. 
48. Udpa N, Zhou D, Haddad GG, Bafna V: Tests of selection in pooled case-control data: an empirical study. Front Genet 2011, 2:83.

49. Holsinger KE, Weir BS: Genetics in geographically structured populations: defining, estimating and interpreting F(ST). Nat Rev Genet 2009, 10:639-650

50. Hudson RR, Slatkin M, Maddison WP: Estimation of levels of gene flow from DNA sequence data. Genetics 1992, 132:583-589.

51. Cavalli-Sforza L: Human diversity. Proc 12th Int Cong Genet 1969, 2:16.

52. Shriver MD, Kennedy GC, Parra EJ, Lawson HA, Sonpar V, Huang J, Akey JM, Jones KW: The genomic distribution of population substructure in four populations using 8,525 autosomal SNPs. Hum Genomics 2004, 1:274-286.

53. Chen H, Patterson N, Reich D: Population differentiation as a test for selective sweeps. Genome Res 2010, 20:393-402.

54. Code used to compute tests of positive selection from Whole Genome Sequencing data (Github). [https://github.com/rronen/selection_stats]

55. Pickrell JK, Coop G, Novembre J, Kudaravalli S, Li JZ, Absher D, Srinivasan BS, Barsh GS, Myers RM, Feldman MW, Pritchard JK: Signals of recent positive selection in a worldwide sample of human populations. Genome Res 2009, 19:826-837.

56. Hudson RR: Generating samples under a Wright-Fisher neutral model of genetic variation. Bioinformatics 2002, 18:337-338

57. Fay JC, Wu Cl: Hitchhiking under positive Darwinian selection. Genetics 2000, 155:1405-1413.

58. Achaz G: Frequency spectrum neutrality tests: one for all and all for one. Genetics 2009, 183:249-258.

doi:10.1186/gb-2014-15-2-r36

Cite this article as: Udpa et al:: Whole genome sequencing of Ethiopian highlanders reveals conserved hypoxia tolerance genes. Genome Biology 2014 15:R36

\section{Submit your next manuscript to BioMed Central and take full advantage of:}

- Convenient online submission

- Thorough peer review

- No space constraints or color figure charges

- Immediate publication on acceptance

- Inclusion in PubMed, CAS, Scopus and Google Scholar

- Research which is freely available for redistribution 